\title{
Cement augmentation of the proximal humerus internal locking system in elderly patients: a multicenter randomized controlled trial
}

\author{
Clemens Hengg ${ }^{1} \cdot$ Stefaan Nijs ${ }^{2}$. Tim Klopfer ${ }^{3} \cdot$ Martin Jaeger $^{4} \cdot$ Andreas Platz $^{5} \cdot$ Tim Pohlemann $^{6} \cdot$ Reto Babst $^{7}$. \\ Jochen Franke ${ }^{8} \cdot$ Franz Kralinger $^{9,10}$
}

Received: 19 September 2018 / Published online: 25 February 2019

(c) The Author(s) 2019

\begin{abstract}
Introduction Cement augmentation of the proximal humerus internal locking system (PHILOS) screws might reduce complication rates in osteoporotic bones. This study compared the risk of mechanical failure during the first year after PHILOS ${ }^{\mathrm{TM}}$ treatment of proximal humerus fractures (PHF) without (control group) and with (augmented group) screw augmentation. Secondary objectives were to report shoulder functions, quality of life (QoL), adverse events (AEs), and reoperation rates. Materials and methods This multicenter randomized trial enrolled patients aged $\geq 65$ years with displaced/unstable PHF from eight European centers. Randomization was performed during surgery through sealed opaque envelopes. Mechanical failures were assessed by two independent reviewers via radiographs, shoulder function by Quick DASH, SPADI, and Constant Murley scores, and QoL by EQ-5D. Follow-ups were planned at postoperative 6 weeks, 3, 6, and 12 months.

Results The preliminary analysis of 6-week radiographs of the first 59 enrolled patients suggested a mechanical failure rate lower than expected and the difference between groups was too small to be detected by the planned sample size of 144 . The trial was prematurely terminated after 67 patients had been enrolled: 34 ( 27 eligible) in the control group and 33 ( 29 eligible) in the augmented group. Follow-ups were performed as planned. Nine patients had mechanical failures and the failure rates (95\% CI) were: augmented group, $16.1 \%(5.5 ; 33.7)$; control group, $14.8 \%(4.2 ; 33.7)$; the relative risk (95\% CI) for the augmented group was $1.09(0.32 ; 3.65)$ compared to the control group $(p=1.000)$. No statistically significant differences in shoulder function, QoL, and AEs were observed between study groups at 1 year. Nine patients (15.8\%) underwent a revision. Conclusions Due to premature termination, the study was underpowered. A larger study will be necessary to determine if cement augmentation lowers the risk of mechanical failure rate.
\end{abstract}

Keywords Proximal humerus fracture $\cdot$ Proximal humerus internal locking system $\cdot$ PHILOS $\cdot$ Augmentation $\cdot$ Osteoporosis · Multicenter randomized trial

Franz Kralinger

franz.kralinger@meduniwien.ac.at

1 Department for Trauma Surgery, A.ö. Landeskrankenhaus - Universitätskliniken Innsbruck, Anichstrasse 35, 6020 Innsbruck, Austria

2 Department of Traumatology, UZ Leuven, Herestraat 49, 3000 Leuven, Belgium

3 Department for Traumatology and Reconstructive Surgery, BG Trauma Center, Eberhard Karls University Tübingen, Schnarrenbergstrasse 95, 72076 Tübingen, Germany

4 Department of Orthopedics and Trauma Surgery, Medical Center-Albert-Ludwigs-University of Freiburg, Hugstetter Strasse 55, 79106 Freiburg, Germany

5 Department of Traumatology, City Hospital Triemli Zurich, Birmensdorferstrasse 497, 8063 Zurich, Switzerland
6 Department of Trauma-, Hand- and Reconstructive Surgery, Saarland University, Kirrberger Strasse, Bldng. 57, 66421 Homburg, Germany

7 Department of Orthopedics and Traumatology, Cantonal Hospital Lucerne, Spitalstrasse, 6000 Lucerne 16, Switzerland

8 Clinic for Trauma and Orthopaedic Surgery, BG Trauma Center Ludwigshafen, Ludwig-Guttmann-Strasse 13, 67071 Ludwigshafen, Germany

9 Medical University of Vienna, Spitalgasse 23, 1090 Vienna, Austria

10 Trauma and Sports Department, Teaching Hospital Medical University of Vienna, Wilhelminenspital, Montlearstr. 37, 1160 Vienna, Austria 


\section{Introduction}

Complex proximal humerus fractures (PHF) often occur in older adults, especially in women, and are oftentimes osteoporotic in nature [1]. The main goal in treating displaced fractures or fracture dislocations is to achieve good clinical shoulder function with no pain via restoration of the proximal humeral anatomy - a goal best achieved by open reduction and internal fixation (ORIF) together with the use of locking plates [2-9]. The proximal humerus internal locking system (PHILOS) has gained wide acceptance and shown good functional outcomes (e.g., assessed using the Constant Murley Score) [10-12]. Nevertheless, even when using PHILOS, the reported complication rates and reoperation frequencies remain high [3, 8, 13-16]. Many of the reported complications were mechanical failures such as loss of reduction and secondary screw perforation, which are believed to be associated with poor anchorage in osteoporotic bone $[17,18]$. Other risk factors include aging, low local bone mineral density (BMD), lack of anatomical reduction, poor restoration of medial cortical support, fracture severity (3- and 4-part fractures), and a varus impacted fracture [3, 4, 7-9, 14, 17-20].

With the aging of the global population, the incidence of osteoporotic fractures is expected to increase. Reduced bone quality presents a challenge to orthopedic surgeries, where complications such as postoperative nonunion, screw cutout, and implant migration, adversely affect patient outcomes [21]. Both Egol et al. and Owsley et al. suggested that age influences the rate of screw cutout in PHF patients [4, 22]. Kralinger et al. reported a mechanical failure rate of $35 \%$ in a prospective multicenter cohort study of 150 patients with displaced PHF fixed with PHILOS [23].

Biomechanical tests of the proximal humerus showed that augmentation of screw tips with polymethylmethacrylate (PMMA) cement improved the mechanical properties of the bone-implant complex, especially in low mineral density bones [24-26]. To date, there has been little clinical evidence supporting the benefit of PMMA augmentation of screw tips [27-29].

The primary objective of the current study was to compare the mechanical failure rates of PHILOS treatment without and with screw augmentation in elderly PHF patients at 1-year post-surgery. Secondary objectives were to compare the study groups regarding shoulder functions, quality of life (QoL), adverse events (AEs), and reoperation rates.

\section{Patients and methods}

\section{Study design and setting}

The present study was a multicenter randomized controlled trial (ClinicalTrials.gov identifier: NCT01847508), with a follow-up period of 1 year after the initial treatment. Patients were enrolled from eight European study centers between January 2014 and April 2016. The last follow-up examination took place in April 2017. Enrolled patients were randomized into two groups. The control group received PHILOS $^{\mathrm{TM}}$ (DePuy Synthes, Oberdorf, Switzerland) without augmentation and the augmented group received PHILOS ${ }^{\mathrm{TM}}$ Augmentation (PHILOS ${ }^{\mathrm{TM}}$ with screw augmentation using Traumacem V + Cement Kit, DePuy Synthes, Oberdorf, Switzerland). Randomization was stratified for each participating center and took place during surgery via opaque sealed envelopes after the fracture reduction was achieved and cannulated locking screws were inserted into the proximal part of the PHILOS ${ }^{\mathrm{TM}}$ plate. Three block sizes were used, with the first block always consisting of six patients and the subsequent blocks of either two or four patients, chosen at random. To maintain allocation concealment, the pattern of the blocks was kept confidential. Patients allocated to the augmented group who failed the leakage test (i.e., with zero or one screw hole suitable for augmentation) received the control treatment and were kept in the study.

The site staff entered all the data into a web-based Electronic Data Capture system, REDCap [30], hosted at the AO Foundation.

\section{Patients}

Patients aged 65 years and older, diagnosed radiographically with an acute ( $\leq 10$ days), closed, displaced or unstable 3- or 4-part PHF sustained after low-energy trauma, and scheduled for primary fracture treatment with a PHILOS $^{\mathrm{TM}}$ plate were included.

Patients with bilateral or previous PHF, cuff-arthropathy on either side, a splitting fracture or an impression fracture of the humeral head, or associated nerve or vessel injuries were excluded. Any known clotting disorders, severe cardiac and/or pulmonary insufficiencies, severe systemic diseases classified as American Society of Anesthesiologists (ASA) class IV to VI, or not medically managed severe systemic diseases classified as ASA class III were also grounds for exclusion. Furthermore, patients with known hypersensitivity or allergy to any of the components of the Traumacem $\mathrm{V}+$ Cement Kit were excluded. Patients were also excluded from this study if they were prisoners, had a recent history of substance abuse (i.e., excessive recreational drugs and/or alcohol consumption) that would preclude reliable assessment, or had participated in any other medical device or medicinal product study within the previous month that could possibly influence the results of the present study.

In addition, patients were excluded before randomization if they received implants other than PHILOS or PHILOS screw augmentation. 


\section{Study treatment}

Surgical treatment was performed as described in the surgical technique guides for PHILOS ${ }^{\mathrm{TM}}$ and PHILOS ${ }^{\mathrm{TM}}$ Augmentation with the use of a deltopectoral approach [31, 32]. Leakage tests were performed in the augmented group by applying a contrast dye to each screw intended to be augmented. If no leakage into the shoulder joint was detected (i.e., negative leakage test), an injection of cement $(\leq 0.5 \mathrm{ml})$ was performed under image intensifier control [24]. In case of leakage into the joint (positive leakage test), an alternative screw was selected for augmentation. To ensure a relatively homogenous effect of augmentation, each patient in the augmented group must have 2-4 screws augmented.

\section{Objectives and endpoints}

Outcomes were evaluated at baseline (before surgery), during surgery, and at 6 weeks, 3 months, 6 months, and 12 months after surgery.

Relevant baseline data including BMD (measured by $\mathrm{CT}$ in the contralateral humeral head) [33] and the comorbidity status (assessed according to the Charlson Comorbidity Index) [34, 35] were assessed before surgery and randomization.

The primary endpoint was the occurrence of mechanical failure during the first year after treatment. Mechanical failures were defined as loss of reduction $\left(\geq 15^{\circ}\right.$ increase of varus malposition and a relative change of $\geq 5 \mathrm{~mm}$ of the greater or lesser tuberosity), humeral head impaction ( $\geq 5 \mathrm{~mm}$ difference in the outer plate edge and tangent of the humeral head), screw/plate loosening (any outward movement of screw position), and secondary screw perforation (perforation of 1 or more screws through the humeral head). The final assessment of mechanical failure for each patient was done after the last follow-up visit by two experienced independent reviewers. Radiographs were taken postoperatively and at each follow-up visit. Follow-up radiographs from each patient were compared with their postoperative radiographs to determine whether mechanical failures had occurred. Disagreement between the reviewers was resolved by consensus. The mechanical failure risk within the first year after treatment was compared between the treatment groups.

Secondary endpoints: Shoulder function was measured by the Quick Disabilities of the Arm, Shoulder and Hand (DASH) measure, the Shoulder Pain and Disability Index (SPADI), and the Constant Murley score [36-43]. Quality of life (QoL) was measured using the EuroQol-5D (EQ-5D) questionnaire. Local and general AEs were recorded for both groups. For the augmented group, the number of incidences with direct contrast fluid leakage into the joint and augmentation-related AEs was also recorded.

Quick DASH, SPADI, and QoL were evaluated at each visit; baseline assessment referred to the pre-injury status. Constant Murley score was assessed at 3, 6, and 12 months after surgery. AEs were recorded from surgery until the end of the final follow-up.

\section{Postoperative care}

All the postoperative treatments were done according to the standard of care at the investigational sites. The end of each patient's postoperative immobilization with a shoulder sling and the start of active range of motion (ROM) were documented.

\section{Statistical analysis/sample size}

The clinical settings and the definitions of mechanical failures of previously reported PHF studies have been quite heterogeneous. The sample size for the current study was calculated based on an earlier prospective multicenter study with a similar design that reported a mechanical failure rate of 35\% [23] in 150 patients aged 50 years or older. Since the current study was designed to include an older patient population ( $\geq 65$ years), the mechanical failure rate for the control group was assumed to be slightly higher and estimated at $40 \%$. Augmentation was hypothesized to reduce the risk of mechanical failure rate to $15 \%$. With a power of $80 \%$, a significance level of 5\%, a 1-year follow-up rate of $\geq 80 \%$, and equal treatment group sizes, the sample size calculation resulted in 144 patients ( 72 per group).

\section{Preliminary analysis}

Due to slow recruitment, a preliminary mechanical failure analysis was done on the first 59 patients. All 6-week follow-up images collected were evaluated by two independent reviewers not involved in the recruitment of patients. The mechanical failure rates were calculated for each study group by an independent statistician. The results showed that the mechanical failure rates of both treatment groups were drastically lower than expected and were very similar to each other. The original sample size was underpowered to detect such a small difference between the two groups; therefore, the study was prematurely terminated after 67 patients had been enrolled. Follow-ups were conducted as planned.

Both intention to treat (ITT) and per protocol (PP) analyses were performed for all outcome parameters. Simple summary statistics were produced for all the outcomes. Comparison of treatment groups was tested at the two-sided 5\% significance level. For the calculation of the risk of mechanical failure, patients with incomplete follow-up and without 
radiographic mechanical failures were excluded. The risk of mechanical failure was calculated as follows:

Number of patients experiencing at least 1 mechanical failure/

(Number of patients completed 1-year follow-up

+ Number of patients with incomplete 1-year follow

-up but had earlier radiographic mechanical failures)

The Clopper-Pearson method was used to calculate the 95\% confidence intervals (CI) for risk of mechanical failure in the two treatment groups. A two-sided Fisher's exact test was used to compare the risk of mechanical failure between the control and the augmented groups. Treatment effects were expressed as relative risks along with their 95\% CI.

Functional outcomes (Constant Murley score, Quick DASH, and SPADI) and QoL (EQ-5D index and EQ-5D VAS) were analyzed using mixed effects models for repeated measures. The models included the fixed categorical effects of treatment, visit and treatment-by-visit interaction, as well as a random effect for study center. An unstructured covariance matrix was used to model the within-patient errors. Models were fitted using restricted maximum likelihood estimation. Significance tests were based on least-squares means.

Time to end of immobilization and time to start with active ROM were analyzed using Kaplan-Meier plots and log-rank tests.

Differences in AE rates between the treatment groups were tested using Fisher's exact test.

\section{Results}

\section{Patient disposition}

The preliminary analysis showed a failure rate of $20 \%$ for the control group instead of the expected $40 \%$, and $12.9 \%$ for the augmented group. Given that the assumptions for the sample size calculation were wrong and the recruitment was very slow, the study was prematurely terminated after 67 patients had given informed consent and were randomized. The control group included 34 patients, of these 27 were eligible; the augmented group had 33 patients, of these 29 were eligible, Fig. 1.

Eleven patients were determined to be ineligible after randomization but were kept in the study. The reasons for ineligibility included: five patients had two-part fractures, one had a two-part fracture and the injury was older than 10 days, two had fractures older than 10 days, one had associated nerve/vessel injury, and two received implants other than PHILOS. Three more patients had protocol violation due to the following reasons: having more than four screws augmented, not having a leakage test performed before augmentation, and/or receiving screw augmentation despite joint perforation. In total, the treatment of 14 patients did not conform to the original protocol. Three patients from the augmented group crossed over to the control group due to positive leakage tests, resulting in 50 patients included for PP analysis.

The follow-up rate at 1 -year was $85.1 \%$ : ten patients (seven from the control and three from the augmented group) dropped out. The reasons for dropping out were either unknown (two patients) or withdrawal of consent (eight patients) (Fig. 1).

\section{Demographics and description of study population}

Of the 67 randomized patients, $55(82.1 \%)$ were women. The mean age was $76.8 \pm 6.8$ (Table 1). All patients except for two from the augmented group sustained their injury due to a fall. Two patients from the augmented group smoked (three and eight cigarettes a day). All the patients were Caucasians and lived at home. The mean BMD was $87.2 \pm 20.1 \mathrm{mg} / \mathrm{cm}^{3}$. The median (range) Charlson Comorbidity score was 0.0 points (0.0-3.0).

Based on the fracture classification of Neer et al. [44], 30 patients $(44.8 \%)$ sustained greater tuberosity three-part fractures and 24 (35.8\%) had greater tuberosity/lesser tuberosity four-part fractures. The augmented group had more four-part fractures (45.5\%) than the control group (26.5\%) (Table 1).

\section{Primary endpoint}

In total, nine patients (Table 2, ITT analysis) had mechanical failures within the first year after treatment. All the patients suffered loss of reduction and some had additional mechanical failures such as humeral head impaction (four patients), screw/plate loosening (one patient), and secondary screw perforation (five patients). No statistically significant differences in the occurrence of mechanical failures were found between the two study groups, neither in the ITT nor the PP analysis ( $p=1.000$ and 0.694 , respectively, Table 2). According to the ITT analysis, the relative risk $(95 \% \mathrm{CI})$ of mechanical failure in the augmented group was $1.09(0.32$; $3.65)$ compared to the control and $1.45(0.37 ; 5.79)$ according to the PP analysis (Table 2). Overall, the number of patients suffering mechanical failures was similar between the two study groups.

\section{Secondary endpoints}

\section{Shoulder function}

No statistically significant differences were detected in the Constant Murley scores (affected shoulder and relative 
Fig. 1 CONSORT flowchart for patient recruitment

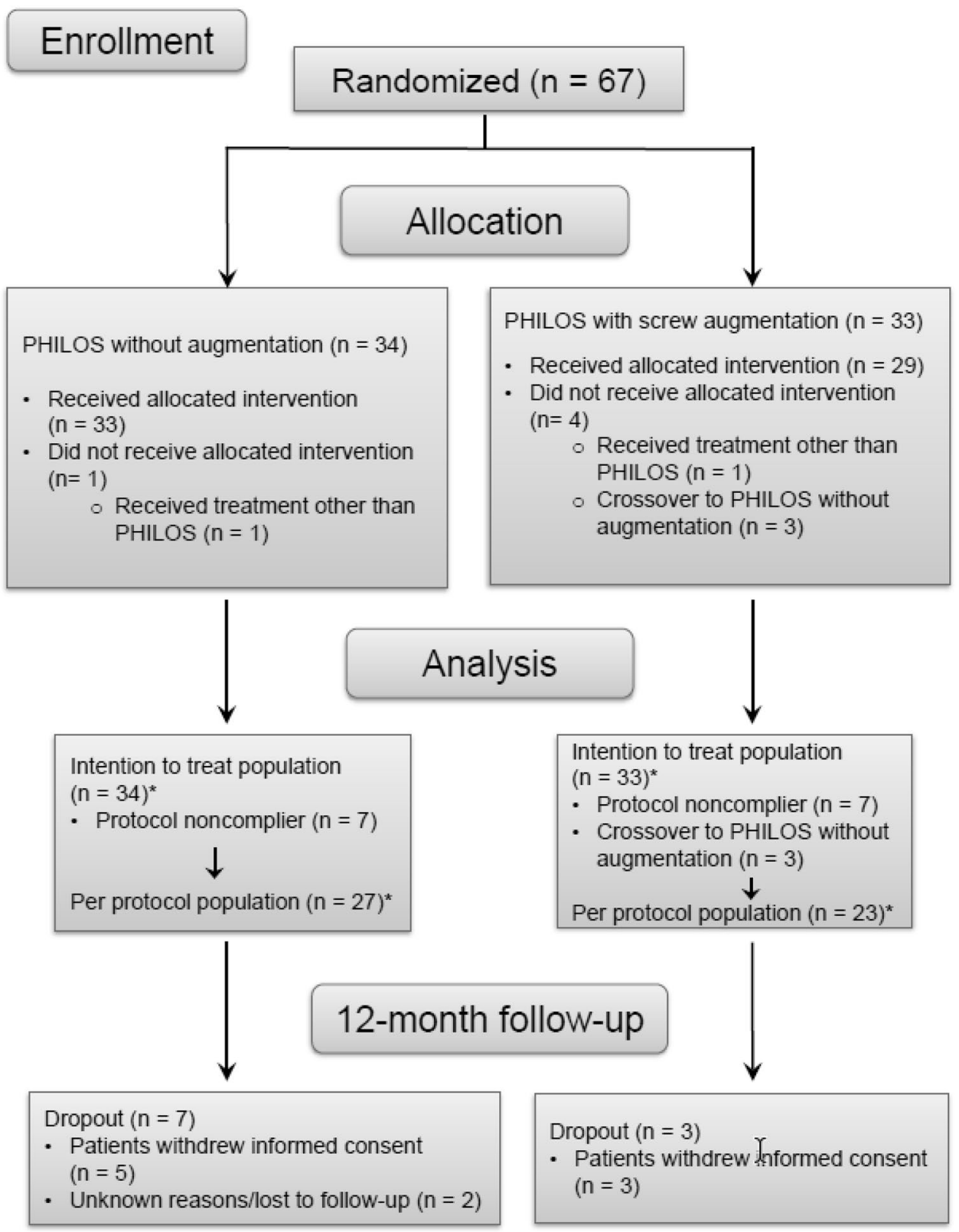

score) between the study groups according to both the ITT and the PP analysis (Table 3). Although baseline Constant Murley scores were not available, the relative Constant Murley scores determined that patients in the control group had reached $78.7 \%$ (95\% CI 69.0; 88.5\%) and in the augmented group, $79.1 \%$ (95\% CI 69.9; 88.3\%), at 12 months after surgery according to the ITT analysis. According to the PP analysis, these were: control group, $83.1 \%$ (95\% CI 73.0; 93.1\%); augmented group, 85.5\% (95\% CI 74.6; 96.4\%).

According to the ITT analyses, no statistically significant differences between the treatment groups were found in the Quick DASH and SPADI scores at any time points except for Quick DASH at 3 months where patients in the control group had better scores than the augmented group (Table 4). The PP analyses, however, showed that patients in the augmented group scored significantly worse than the control group in the Quick DASH evaluation at baseline [mean $(95 \% \mathrm{CI})=11.3(6.6 ; 15.9)$ versus $4.9(0.5 ; 9.2)$, respectively, $p=0.050]$, 6 weeks [mean $(95 \% \mathrm{CI})=56.8(49.6 ; 64.0)$ versus 45.9 (38.7; $53.1)$, respectively, $p=0.037$ ], and 3 months [mean $(95 \%$ $\mathrm{CI})=44.0(35.0 ; 53.0)$ versus $31.0(22.7 ; 39.3)$, respectively, $p=0.038$ ] (Table 5). By 6 months and 1 year after surgery, the augmented group still had higher mean scores, but the differences were not statistically significant. Differences between treatment groups in SPADI scores were not statistically significant according to the PP analyses. 
Table 1 Summary of patient demographics and clinical characteristics (ITT analysis)

\begin{tabular}{|c|c|c|c|}
\hline Characteristic & $\begin{array}{l}\text { PHILOS without } \\
\text { augmentation } N=34\end{array}$ & $\begin{array}{l}\text { PHILOS with screw } \\
\text { augmentation } N=33\end{array}$ & Total $N=67$ \\
\hline Gender, $n(\%)$ & 34 & 33 & 67 \\
\hline Female & $29(85.3)$ & $26(78.8)$ & $55(82.1)$ \\
\hline Male & $5(14.7)$ & $7(21.2)$ & $12(17.9)$ \\
\hline \multicolumn{4}{|l|}{ Age (years) } \\
\hline$n$ & 34 & 33 & 67 \\
\hline Mean (SD) & $76.1(6.2)$ & $77.5(7.4)$ & $76.8(6.8)$ \\
\hline Median (min; max) & $76.0(65.0 ; 90.0)$ & $80.0(65.0 ; 92.0)$ & $77.0(65.0 ; 92.0)$ \\
\hline Smoker, $n(\%)$ & 34 & 33 & 67 \\
\hline No & $34(100.0)$ & $31(93.9)$ & $65(97.0)$ \\
\hline Yes & $0(0.0)$ & $2(6.1)$ & $2(3.0)$ \\
\hline \multicolumn{4}{|c|}{ Bone mineral density mean $\left(\mathrm{mg} / \mathrm{cm}^{3}\right)$} \\
\hline$n$ & 28 & 27 & 55 \\
\hline Mean (SD) & $88.1(21.8)$ & $86.1(18.6)$ & $87.2(20.1)$ \\
\hline Median (min; max) & $86.7(42.7 ; 148.4)$ & $82.0(51.1 ; 128.9)$ & $84.5(42.7 ; 148.4)$ \\
\hline Mechanism of injury, $n(\%)$ & 34 & 33 & 67 \\
\hline Fall & $34(100.0)$ & $31(93.9)$ & $65(97.0)$ \\
\hline Other & $0(0.0)$ & $2(6.1)$ & $2(3.0)$ \\
\hline Neer classification, $n(\%)$ & 34 & 33 & 67 \\
\hline Anatomical neck two-part & $1(2.9)$ & $0(0.0)$ & $1(1.5)$ \\
\hline Surgical neck two-part & $3(8.8)$ & $1(3.0)$ & $4(6.0)$ \\
\hline Greater tuberosity two-part & $1(2.9)$ & $0(0.0)$ & $1(1.5)$ \\
\hline Greater tuberosity three-part & $15(44.1)$ & $15(45.5)$ & $30(44.8)$ \\
\hline Lesser tuberosity two-part & $0(0.0)$ & $0(0.0)$ & $0(0.0)$ \\
\hline Lesser tuberosity three-part & $2(5.9)$ & $1(3.0)$ & $3(4.5)$ \\
\hline Four-part & $9(26.5)$ & $15(45.5)$ & $24(35.8)$ \\
\hline Anterior two-part & $0(0.0)$ & $0(0.0)$ & $0(0.0)$ \\
\hline Anterior three-part & $0(0.0)$ & $0(0.0)$ & $0(0.0)$ \\
\hline Anterior four-part & $0(0.0)$ & $0(0.0)$ & $0(0.0)$ \\
\hline Anterior articular surface & $0(0.0)$ & $0(0.0)$ & $0(0.0)$ \\
\hline Posterior two-part & $0(0.0)$ & $0(0.0)$ & $0(0.0)$ \\
\hline Posterior three-part & $0(0.0)$ & $0(0.0)$ & $0(0.0)$ \\
\hline Posterior four-part & $3(8.8)$ & $1(3.0)$ & $4(6.0)$ \\
\hline Posterior articular surface & $0(0.0)$ & $0(0.0)$ & $0(0.0)$ \\
\hline Head-splitting articular surface & $0(0.0)$ & $0(0.0)$ & $0(0.0)$ \\
\hline \multicolumn{4}{|l|}{ Charlson comorbidity index ${ }^{a}$} \\
\hline$n$ & 34 & 33 & 67 \\
\hline Mean (SD) & $0.6(0.9)$ & $0.5(0.8)$ & $0.6(0.8)$ \\
\hline Median (min; max) & $0.0(0.0 ; 3.0)$ & $0.0(0.0 ; 2.0)$ & $0.0(0.0 ; 3.0)$ \\
\hline
\end{tabular}

ITT intention to treat, PHILOS Proximal humerus internal locking system

a The minimum possible score is 0 and the maximum possible score is 29 . A higher score indicates a greater burden of comorbid conditions
Patients from both groups showed improvement in their Constant Murley, Quick DASH and SPADI scores throughout the follow-up period. However, the mean scores at 12 months were still significantly worse than the mean scores at baseline in Quick DASH according to the ITT but not the PP analysis [control group mean $=11.7(95 \%$
CI $1.6 ; 21.8)$ at 12 months versus $4.9(0.5 ; 9.2)$ at baseline, $p=0.172$, and augmented group mean $=18.8(8.0 ; 29.6)$ at 12 months versus $11.3(6.6 ; 15.9)$ at baseline, $p=0.157]$. Mean SPADI scores at 12 months were significantly worse than scores at baseline for both treatment groups according to both analyses (Tables 4, 5). 
Table 2 Mechanical failure occurrence within 1 year after surgery according to radiological review

\begin{tabular}{|c|c|c|c|c|c|c|}
\hline & \multicolumn{2}{|c|}{$\begin{array}{l}\text { PHILOS without aug- } \\
\text { mentation }\end{array}$} & \multicolumn{2}{|c|}{$\begin{array}{l}\text { PHILOS with screw } \\
\text { augmentation }\end{array}$} & \multirow{2}{*}{$\begin{array}{l}\text { Relative risk }{ }^{\mathrm{c}} \\
\left(95 \% \mathrm{CI}^{\mathrm{d}}\right)\end{array}$} & \multirow[t]{2}{*}{$p$ value $\mathrm{e}^{\mathrm{e}}$} \\
\hline & $n / N$ & $\%\left(95 \% \mathrm{CI}^{\mathrm{b}}\right)$ & $n / N$ & $\%\left(95 \% \mathrm{CI}^{\mathrm{b}}\right)$ & & \\
\hline Intention to treat analysis ${ }^{\mathrm{a}}$ & $4 / 27$ & $14.8(4.2 ; 33.7)$ & $5 / 31$ & $16.1(5.5 ; 33.7)$ & $1.09(0.32 ; 3.65)$ & 1.000 \\
\hline Per protocol analysis ${ }^{\mathrm{a}}$ & $3 / 24$ & $12.5(2.7 ; 32.4)$ & $4 / 22$ & $18.2(5.2 ; 40.3)$ & $1.45(0.37 ; 5.79)$ & 0.694 \\
\hline
\end{tabular}

PHILOS Proximal humerus internal locking system

${ }^{\text {a } P a t i e n t s ~ w i t h o u t ~ r a d i o g r a p h s ~(i n ~ a t ~ l e a s t ~ t w o ~ p l a n e s) ~ f o r ~ t h e ~ 1-y e a r ~ f o l l o w-u p ~ w e r e ~ c o u n t e d ~ a s ~ h a v i n g ~ m i s s-~}$ ing values, unless they had a radiographically assessed mechanical failure at an earlier time point

${ }^{b}$ Confidence intervals for percentages were calculated using the Clopper-Pearson method

${ }^{c}$ Relative risk comparing PHILOS with augmentation against PHILOS without augmentation

${ }^{\mathrm{d}}$ Confidence interval was calculated using the Wald method

${ }^{\mathrm{e}}$ Fisher's exact test

Table 3 Constant Murley Score (affected shoulder) and relative Constant Murley score over the course of follow-up, mixed effects model analysis

\begin{tabular}{|c|c|c|c|c|c|}
\hline Intention to treat analysis & $n$ & $\begin{array}{l}\text { PHILOS without aug- } \\
\text { mentation } \\
N=34 \\
\text { Mean }(95 \% \mathrm{CI})\end{array}$ & $n$ & $\begin{array}{l}\text { PHILOS with augmentation } \\
N=33 \\
\text { Mean }(95 \% \text { CI })\end{array}$ & $p$ value $^{\mathrm{c}}$ \\
\hline \multicolumn{6}{|l|}{ Constant Murley score ${ }^{a}$} \\
\hline 3 months & 23 & $45.7(38.2 ; 53.3)$ & 20 & $40.5(32.6 ; 48.4)$ & 0.315 \\
\hline 6 months & 23 & $58.7(51.4 ; 65.9)$ & 22 & $55.6(48.4 ; 62.8)$ & 0.530 \\
\hline 12 months & 23 & $66.6(58.7 ; 74.6)$ & 27 & $64.4(56.8 ; 71.9)$ & 0.665 \\
\hline \multicolumn{6}{|c|}{ Relative Constant Murley score ${ }^{\mathrm{b}}$} \\
\hline 3 months & 21 & $57.0(46.7 ; 67.2)$ & 19 & $53.4(43.1 ; 63.8)$ & 0.612 \\
\hline 6 months & 23 & $71.7(62.5 ; 80.9)$ & 22 & $69.6(60.6 ; 78.6)$ & 0.726 \\
\hline 12 months & 23 & $78.7(69.0 ; 88.5)$ & 27 & $79.1(69.9 ; 88.3)$ & 0.954 \\
\hline Per protocol analysis & $n$ & $\begin{array}{l}\text { PHILOS without aug- } \\
\text { mentation } \\
N=27 \\
\text { Mean (95\% CI) }\end{array}$ & $n$ & $\begin{array}{l}\text { PHILOS with augmentation } \\
N=23 \\
\text { Mean }(95 \% \text { CI })\end{array}$ & $p$ value $^{\mathrm{c}}$ \\
\hline \multicolumn{6}{|l|}{ Constant Murley score ${ }^{\mathrm{a}}$} \\
\hline 3 months & 17 & $45.0(36.3 ; 53.7)$ & 12 & $45.8(35.6 ; 56.0)$ & 0.900 \\
\hline 6 months & 16 & $60.7(53.4 ; 68.1)$ & 15 & $55.6(47.7 ; 63.6)$ & 0.342 \\
\hline 12 months & 15 & $69.8(62.5 ; 77.2)$ & 12 & $69.9(61.7 ; 78.0)$ & 0.996 \\
\hline \multicolumn{6}{|c|}{ Relative Constant Murley score ${ }^{\mathrm{b}}$} \\
\hline 3 months & 16 & $54.4(42.2 ; 66.6)$ & 12 & $57.1(43.8 ; 70.5)$ & 0.750 \\
\hline 6 months & 16 & $74.3(64.1 ; 84.6)$ & 15 & $69.8(59.2 ; 80.5)$ & 0.510 \\
\hline 12 months & 15 & $83.1(73.0 ; 93.1)$ & 12 & $85.5(74.6 ; 96.4)$ & 0.727 \\
\hline
\end{tabular}

Results are from a mixed effects model for repeated measures with an unstructured covariance (patient level) and a random center effect

PHILOS Proximal humerus internal locking system

${ }^{a}$ The Constant Murley score assesses shoulder function and pain in patients after shoulder injuries and various shoulder treatments. It ranges from 2 (worst) to 100 (best)

${ }^{\mathrm{b}}$ Operated compared to contralateral shoulder, in \%

${ }^{c} p$ values comparing mean values between the treatment groups at the specified time points

\section{Quality of life}

According to the ITT analyses, no statistical differences in the EQ-5D index nor the EQ-VAS health state scores were detected between the two treatment groups at any time point
(Table 4). PP analyses also revealed no statistical differences for all time points, although the 6 weeks EQ-5D index $p$ value $(p=0.051)$ and the 6 weeks and 12 months EQ-5D VAS $p$ values ( $p=0.054$ and 0.055 , respectively) were borderline significant (Table 5). 


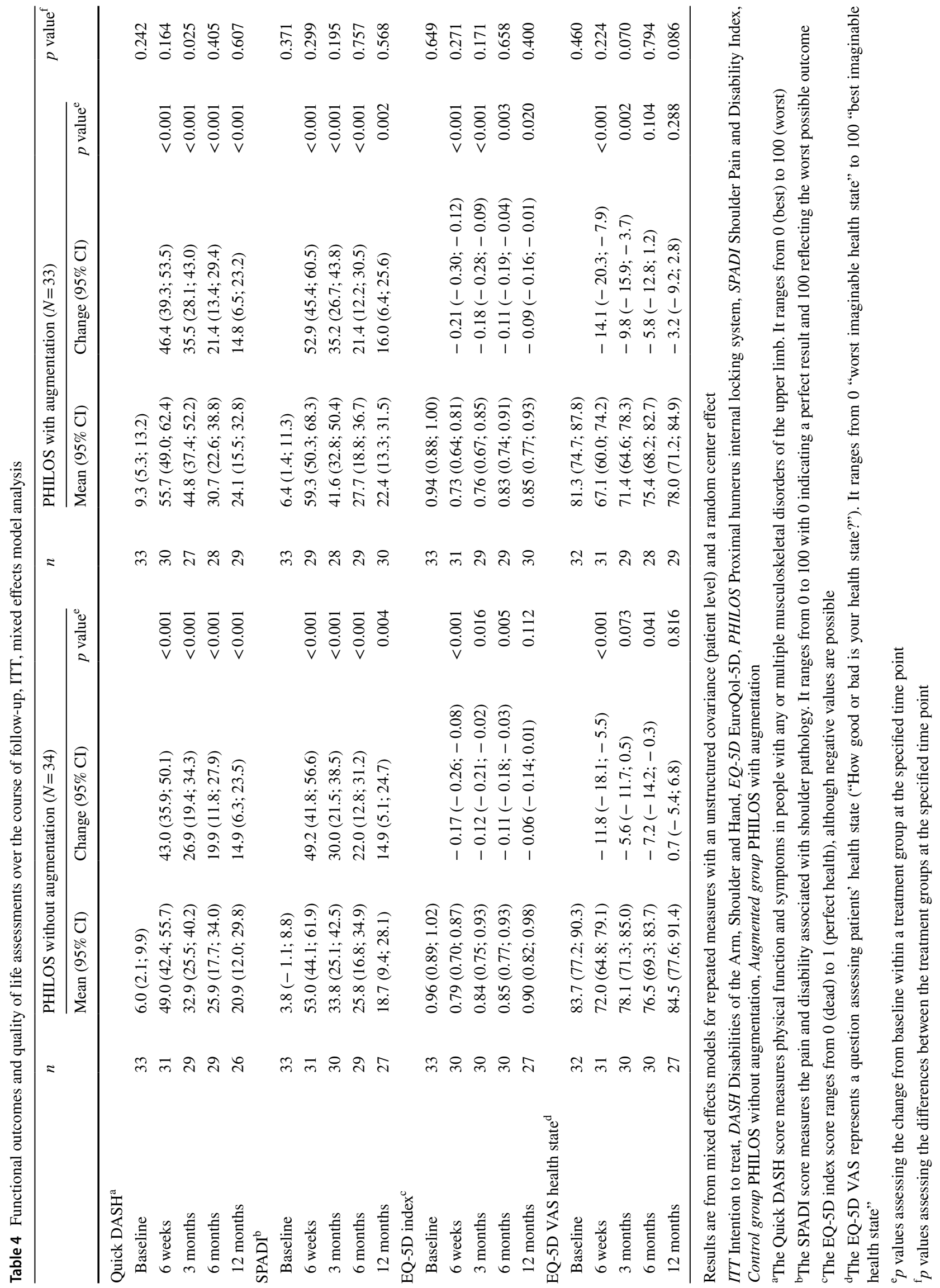




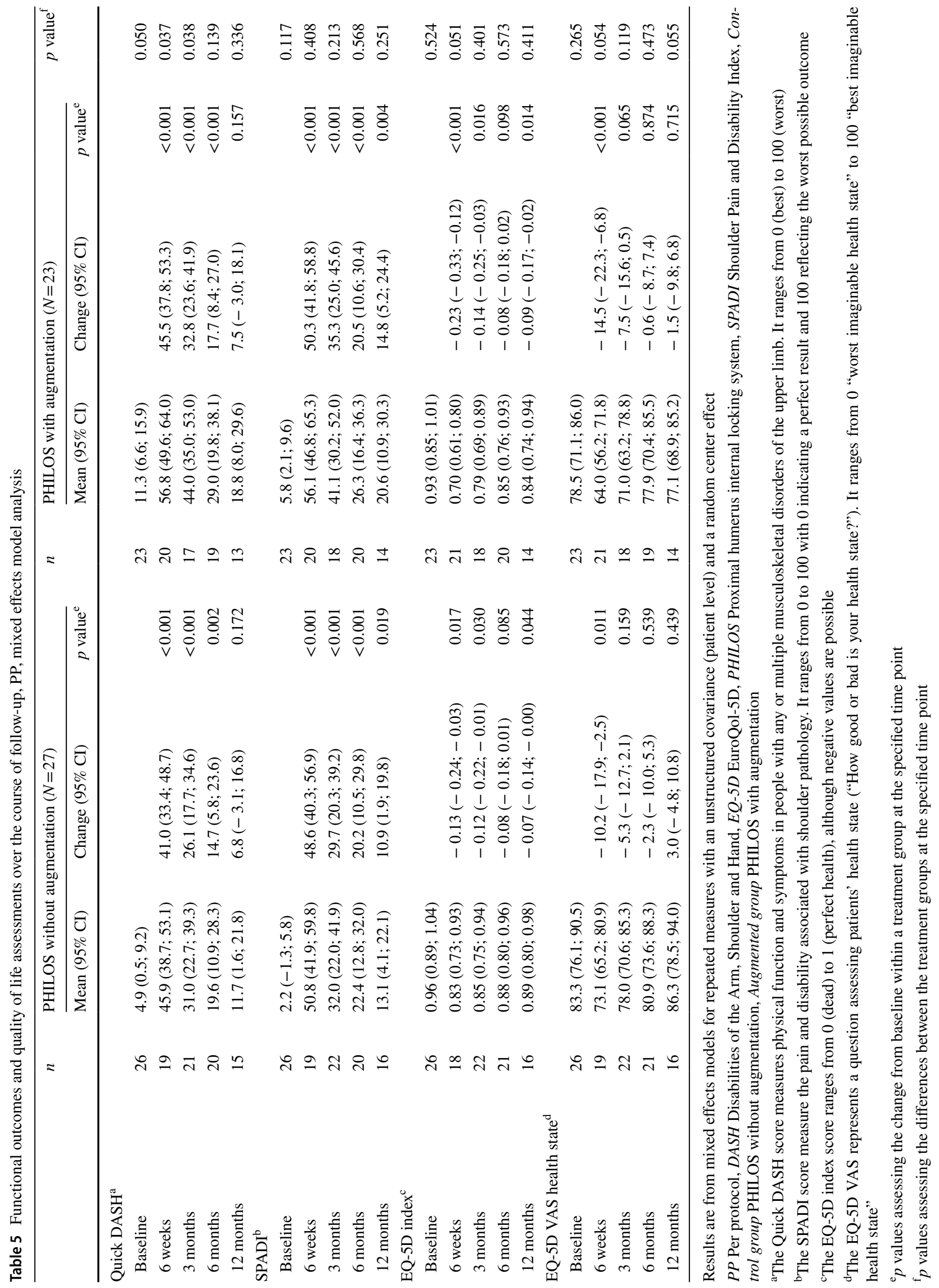




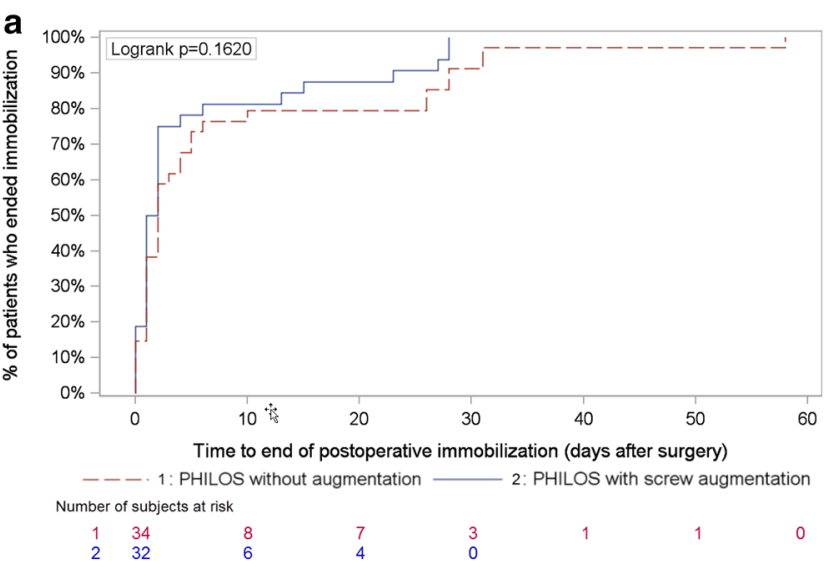

C

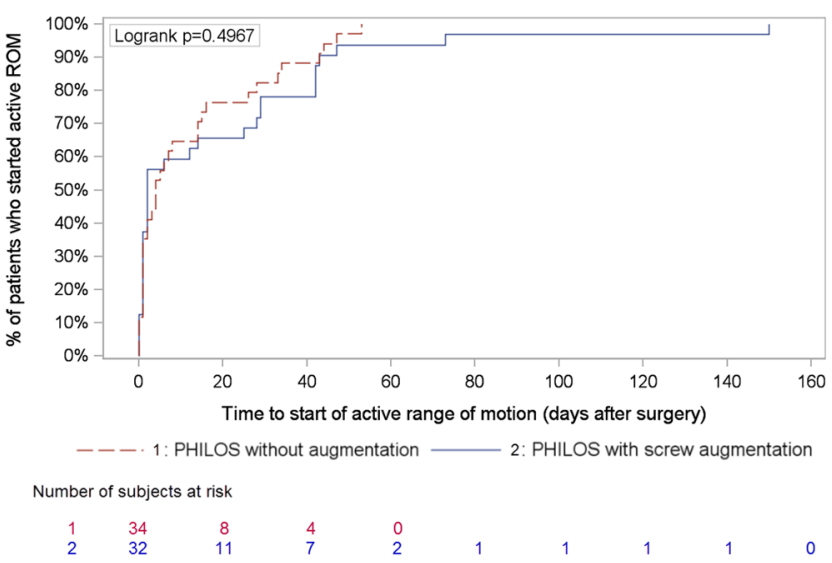

Fig. 2 Postoperative shoulder immobilization and time to active range of motion: Kaplan-Meier analyses of number of subjects at risk. a Time to end of shoulder immobilization (ITT). b Time to end of shoulder immobilization (PP). c Time to start active range of motion

Compared to the baseline values, the EQ-5D index score for the control group was significantly worse at 12 months according to the PP analysis [mean $(95 \% \mathrm{CI})=0.89(0.80 ; 0.98)$ at 12 months versus $0.96(0.89 ; 1.04)$ at baseline, $p=0.044]$ (Table 5), but not according to the ITT analysis [mean (95\% $\mathrm{CI})=0.90(0.82 ; 0.98)$ at 12 months versus $0.96(0.89 ; 1.02)$ at baseline, $p=0.112$ ] (Table 4 ), whereas for the augmented group, the score at 12 months was significantly lower than baseline in both analyses ( $p=0.020$ and $p=0.014$, Tables 4,5$)$.

No statistically significant difference in the EQ-5D VAS scores was detected in either treatment group (according to both the ITT and PP analyses) 1 year after surgery compared to baseline (Tables 4, 5).

\section{Postoperative shoulder immobilization and time to active range of motion}

The median (range) time of postoperative shoulder immobilization was 2.0 days ( 0 to 58 ) in the control group and 2.0 days ( 0 to 28 ) in the augmented group. The time to reach

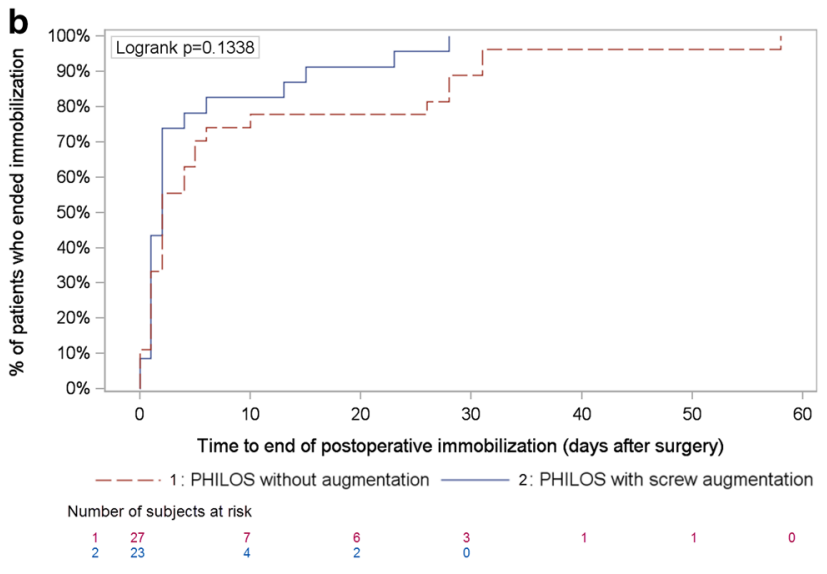

d

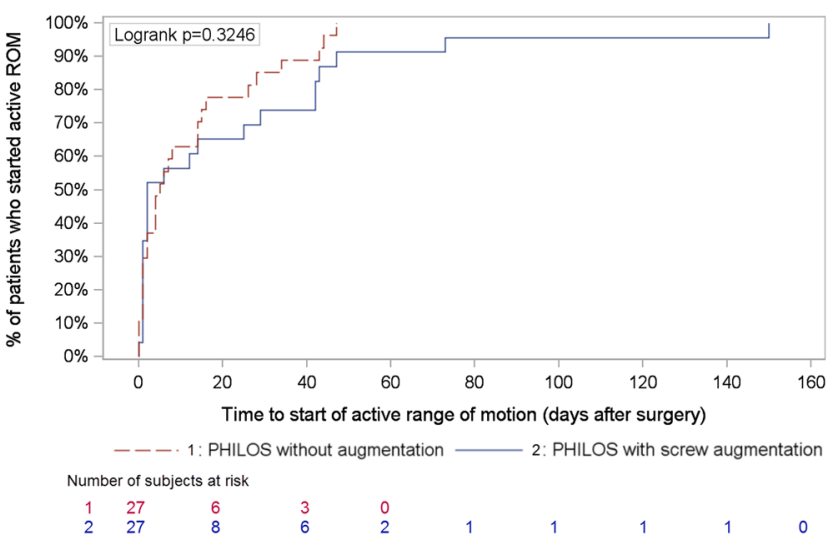

(ITT). d Time to start active range of motion (PP). ITT: intention to treat analysis, PP: Per protocol analysis. Control group: PHILOS without augmentation. Augmented group: PHILOS with augmentation

the end of immobilization did not differ significantly between the two groups according to ITT analyses $(p=0.162)$ and PP analyses ( $p=0.134$ ) (Fig. 2a, b, respectively).

Patients in the control group started with active ROM at a median (range) of 4.0 days (0-53), and in the augmented group, 2.0 days $(0-150)$. There were no significant differences between the two groups ( $p=0.497$, ITT analyses; $p=0.325$, PP analyses) (Fig. 2c, d, respectively).

\section{Radiological outcomes}

Radiological outcomes were analyzed using the safety population, defined as all patients who received the PHILOS ${ }^{\mathrm{TM}}$ treatment. Anatomical reduction was achieved in 24/35 patients $(68.6 \%)$ of the control group (1 missing value due to the lack of postoperative radiograph) and in 20/29 patients $(69.0 \%)$ of the augmented group; there was no statistically significant difference between the groups $(p=0.973)$. Restoration of medial support was achieved in $22 / 35$ patients (62.9\%) of the control group and in $18 / 29$ patients $(62.1 \%)$ 
of the augmented group, also with no difference between the groups $(p=0.948)$.

\section{Safety analysis}

Adverse events were also analyzed using the safety population. Fifteen patients in the control group and 12 patients in the augmented group reported at least $1 \mathrm{AE}$ during the study (Table 6), resulting in an overall AE rate (95\% CI) of $41.5 \%$ $(29.4 ; 54.4)$. There was no statistically significant difference in the overall $\mathrm{AE}$ reporting rate between the control group, $41.7 \%$ (25.5; 59.2), and the augmented group, 41.4 (23.5; 61.1) $(p=1.000)$.

In total, nine patients underwent revision: one patient had two revision surgeries, first due to hematoma and later, screw perforation. The remaining revision surgeries were due to humeral head necrosis (four patients), humeral head impaction (one patient), loss of reduction (one patient), infection, and malposition of plate/screw (one patient each). Three patients were revised with anatomic prothesis and two with reverse arthroplasty.

\section{Discussion}

The present study aimed to investigate the difference in the risk of mechanical failure in elderly PHF patients treated with PHILOS $^{\mathrm{TM}}$ with or without cement augmentation of the screw tips within the first year after surgery. As recruitment was slow and a preliminary analysis showed a much lower mechanical failure rate than expected with no apparent differences between the groups, the study was prematurely terminated after enrolling less than half the intended number of patients.

The study results showed no statistically significant differences in the mechanical failure rates between study groups at 1 year. Given the early termination of the recruitment, the current study was underpowered. All the estimates of treatment effect with our final sample size had a high uncertainty. The relative risks $(95 \% \mathrm{CI})$ of having a mechanical failure for the augmented group compared to the control group were estimated at $1.09(0.32 ; 3.65)$ in the ITT analysis and 1.45 $(0.37 ; 5.09)$ in the PP analysis. Large confidence intervals (including " 1 ") indicate that the evidence for this study is not sufficient, and a much larger sample size would be required to show a possible difference between the use of PHILOS ${ }^{\text {TM }}$ without augmentation and PHILOS ${ }^{\mathrm{TM}}$ with augmentation in proximal humerus fractures.

Neither ITT nor PP analyses showed statistically significant differences between the treatment groups in shoulder function measured with Constant Murley score, Quick DASH, and SPADI at 1 year. PP analyses with mixed effects model for the Quick DASH showed that the augmented group had statistically significant worse scores than the control group at the initial time points (baseline, 6 weeks, and 3 months after surgery). Although the mean scores for the augmented group remained worse at 6 and 12 months after surgery compared to the control group, the differences were not statistically significant. Several potential explanations exist for this difference. One possibility is the higher proportion of patients with fourpart fracture in the augmented group $(45.5 \%$ versus $26.5 \%$ in the control, ITT analysis), as it has been suggested that patients with four-part fractures tend to have lower functional outcomes than those with two- and three-part fractures [15]. These results could also suggest that the impact of fracture severity on shoulder function after treatment with PHILOS is only temporary since the differences were not significant by 6 months.

Overall, results showed that the injured shoulders continued to recover through the first year after surgery. Even then, shoulder function at 1 year was still worse in both treatment groups compared to baseline. In comparison to 1-year (QUICK) DASH and Constant Murley score results reported in the literature, the current patient population reached similar recovery at 1 year $[4,12,14,20,45,46]$. However, direct comparison of results from different studies may be misleading due their heterogeneity. For example, in the study reported by Gracitelli et al., the patients had two- and three-part PHFs while the current patients had three- or four-part PHF [46]. According to the relative Constant Murley score results, the shoulder function outcome of the current population reached the "good" category according to the PP analysis or the highend of the "satisfactory" category according to the ITT analysis at 12 months postoperatively [excellent (90-100\%), good (80-89\%), satisfactory $(60-79 \%)$, and poor $(<60 \%)][46,47]$.

Similar to the results of shoulder function, there were no statistically significant differences between the treatment groups in QoL at 1 year after surgery (both ITT and PP analysis), although borderline significance was observed at 6 weeks in EQ-5D index scores and 6 weeks and 12 months in EQ-5D VAS scores (PP analysis).

Screw augmentation has been shown to be effective in biomechanical studies, but clinical evidence has been lacking. A recent study by Katthagen et al. comparing 24 patients treated with PHILOS screw augmentation to a historical cohort showed that screw augmentation reduced the likelihood of early loss of reduction and articular screw perforation but resulted in no differences in shoulder function scores [27]. In contrast, the current study showed no overall differences between the treatment groups. Differences between the two studies include: (1) the current study assessed loss of reduction, humeral head impaction, screw/plate loosening, and secondary screw perforation, while the previous study reported only loss of reduction and articular screw perforation. (2) The current study was a prospective study, while the previous study was retrospective with a matched historical cohort. (3) The current study had a follow-up 
Table 6 Summary of adverse events (patient level) within 1 year after surgery (safety population)

\begin{tabular}{|c|c|c|c|c|c|c|c|}
\hline \multirow[t]{2}{*}{ Adverse events $^{\mathrm{a}}$} & \multicolumn{2}{|c|}{$\begin{array}{l}\text { PHILOS without } \\
\text { augmentation } N=36\end{array}$} & \multicolumn{2}{|c|}{$\begin{array}{l}\text { PHILOS with screw } \\
\text { augmentation } N=29\end{array}$} & \multicolumn{2}{|c|}{ Total $N=65$} & \multirow[t]{2}{*}{$p$ value $^{\mathrm{e}}$} \\
\hline & $N^{\mathrm{b}}$ & $\%^{\mathrm{c}}\left(95 \% \mathrm{CI}^{\mathrm{d}}\right)$ & $N^{\mathrm{b}}$ & $\%^{\mathrm{c}}\left(95 \% \mathrm{CI}^{\mathrm{d}}\right)$ & $N^{b}$ & $\%^{\mathrm{c}}\left(95 \% \mathrm{CI}^{\mathrm{d}}\right)$ & \\
\hline Any adverse events & 15 & $41.7(25.5 ; 59.2)$ & 12 & $41.4(23.5 ; 61.1)$ & 27 & $41.5(29.4 ; 54.4)$ & 1.000 \\
\hline Any intraoperative adverse event & 4 & $11.1(3.1 ; 26.1)$ & 1 & $3.4(0.1 ; 17.8)$ & 5 & $7.7(2.5 ; 17.0)$ & 0.370 \\
\hline Poor intraoperative fracture reduction & 3 & $8.3(1.8 ; 22.5)$ & 0 & $0.0(0.0 ; 11.9)$ & 3 & $4.6(1.0 ; 12.9)$ & - \\
\hline Malpositioning of the plate/screw(s) & 2 & $5.6(0.7 ; 18.7)$ & 0 & $0.0(0.0 ; 11.9)$ & 2 & $3.1(0.4 ; 10.7)$ & - \\
\hline Cement leakage into the joint & 0 & $0.0(0.0 ; 9.7)$ & 1 & $3.4(0.1 ; 17.8)$ & 1 & $1.5(0.0 ; 8.3)$ & - \\
\hline Cement application into the wound & 0 & $0.0(0.0 ; 9.7)$ & 0 & $0.0(0.0 ; 11.9)$ & 0 & $0.0(0.0 ; 5.5)$ & - \\
\hline Direct allergic reaction to Traumacem $V+$ cement & 0 & $0.0(0.0 ; 9.7)$ & 0 & $0.0(0.0 ; 11.9)$ & 0 & $0.0(0.0 ; 5.5)$ & - \\
\hline Direct allergic reaction to contrast media & 0 & $0.0(0.0 ; 9.7)$ & 0 & $0.0(0.0 ; 11.9)$ & 0 & $0.0(0.0 ; 5.5)$ & - \\
\hline Implant failure/breakage & 0 & $0.0(0.0 ; 9.7)$ & 0 & $0.0(0.0 ; 11.9)$ & 0 & $0.0(0.0 ; 5.5)$ & - \\
\hline Other bone/fracture-related $\mathrm{AE}$ & 0 & $0.0(0.0 ; 9.7)$ & 0 & $0.0(0.0 ; 11.9)$ & 0 & $0.0(0.0 ; 5.5)$ & - \\
\hline Nerve injury & 0 & $0.0(0.0 ; 9.7)$ & 0 & $0.0(0.0 ; 11.9)$ & 0 & $0.0(0.0 ; 5.5)$ & - \\
\hline Other AE related to soft tissue of the musculoskeletal system & 0 & $0.0(0.0 ; 9.7)$ & 0 & $0.0(0.0 ; 11.9)$ & 0 & $0.0(0.0 ; 5.5)$ & - \\
\hline Other wound/local tissue-related AE & 0 & $0.0(0.0 ; 9.7)$ & 0 & $0.0(0.0 ; 11.9)$ & 0 & $0.0(0.0 ; 5.5)$ & - \\
\hline Thromboembolic complications & 0 & $0.0(0.0 ; 9.7)$ & 0 & $0.0(0.0 ; 11.9)$ & 0 & $0.0(0.0 ; 5.5)$ & - \\
\hline Renal insufficiency & 0 & $0.0(0.0 ; 9.7)$ & 0 & $0.0(0.0 ; 11.9)$ & 0 & $0.0(0.0 ; 5.5)$ & - \\
\hline Stroke & 0 & $0.0(0.0 ; 9.7)$ & 0 & $0.0(0.0 ; 11.9)$ & 0 & $0.0(0.0 ; 5.5)$ & - \\
\hline Sudden death & 0 & $0.0(0.0 ; 9.7)$ & 0 & $0.0(0.0 ; 11.9)$ & 0 & $0.0(0.0 ; 5.5)$ & - \\
\hline Intraoperative hemodynamically relevant hemorrhage & 0 & $0.0(0.0 ; 9.7)$ & 0 & $0.0(0.0 ; 11.9)$ & 0 & $0.0(0.0 ; 5.5)$ & - \\
\hline Other systemic $\mathrm{AE}$ & 0 & $0.0(0.0 ; 9.7)$ & 0 & $0.0(0.0 ; 11.9)$ & 0 & $0.0(0.0 ; 5.5)$ & - \\
\hline Any postoperative adverse event & 14 & $38.9(23.1 ; 56.5)$ & 12 & $41.4(23.5 ; 61.1)$ & 26 & $40.0(28.0 ; 52.9)$ & 1.000 \\
\hline $\begin{array}{l}\text { Late/development of allergic reaction to Traumacem } \\
\mathrm{V}+\text { cement }\end{array}$ & 0 & $0.0(0.0 ; 9.7)$ & 0 & $0.0(0.0 ; 11.9)$ & 0 & $0.0(0.0 ; 5.5)$ & - \\
\hline Late/development of allergic reaction to contrast media & 0 & $0.0(0.0 ; 9.7)$ & 0 & $0.0(0.0 ; 11.9)$ & 0 & $0.0(0.0 ; 5.5)$ & - \\
\hline Primary/secondary screw perforation & 3 & $8.3(1.8 ; 22.5)$ & 1 & $3.4(0.1 ; 17.8)$ & 4 & $6.2(1.7 ; 15.0)$ & - \\
\hline Screw/plate loosening & 0 & $0.0(0.0 ; 9.7)$ & 0 & $0.0(0.0 ; 11.9)$ & 0 & $0.0(0.0 ; 5.5)$ & - \\
\hline Implant failure/breakage & 0 & $0.0(0.0 ; 9.7)$ & 0 & $0.0(0.0 ; 11.9)$ & 0 & $0.0(0.0 ; 5.5)$ & - \\
\hline Nonunion & 0 & $0.0(0.0 ; 9.7)$ & 0 & $0.0(0.0 ; 11.9)$ & 0 & $0.0(0.0 ; 5.5)$ & - \\
\hline Delayed union & 0 & $0.0(0.0 ; 9.7)$ & 0 & $0.0(0.0 ; 11.9)$ & 0 & $0.0(0.0 ; 5.5)$ & - \\
\hline Malunion & 0 & $0.0(0.0 ; 9.7)$ & 1 & $3.4(0.1 ; 17.8)$ & 1 & $1.5(0.0 ; 8.3)$ & - \\
\hline Loss of reduction & 2 & $5.6(0.7 ; 18.7)$ & 1 & $3.4(0.1 ; 17.8)$ & 3 & $4.6(1.0 ; 12.9)$ & - \\
\hline Humeral head necrosis & 2 & $5.6(0.7 ; 18.7)$ & 3 & $10.3(2.2 ; 27.4)$ & 5 & $7.7(2.5 ; 17.0)$ & - \\
\hline Head impaction & 1 & $2.8(0.1 ; 14.5)$ & 0 & $0.0(0.0 ; 11.9)$ & 1 & $1.5(0.0 ; 8.3)$ & - \\
\hline Other bone/fracture-related AE & 1 & $2.8(0.1 ; 14.5)$ & 2 & $6.9(0.8 ; 22.8)$ & 3 & $4.6(1.0 ; 12.9)$ & - \\
\hline Deep wound infection & 0 & $0.0(0.0 ; 9.7)$ & 1 & $3.4(0.1 ; 17.8)$ & 1 & $1.5(0.0 ; 8.3)$ & - \\
\hline Impingement & 2 & $5.6(0.7 ; 18.7)$ & 0 & $0.0(0.0 ; 11.9)$ & 2 & $3.1(0.4 ; 10.7)$ & - \\
\hline Nerve injury & 0 & $0.0(0.0 ; 9.7)$ & 1 & $3.4(0.1 ; 17.8)$ & 1 & $1.5(0.0 ; 8.3)$ & - \\
\hline Other AE related to soft tissue of the musculoskeletal system & 1 & $2.8(0.1 ; 14.5)$ & 2 & $6.9(0.8 ; 22.8)$ & 3 & $4.6(1.0 ; 12.9)$ & - \\
\hline Superficial wound infection & 0 & $0.0(0.0 ; 9.7)$ & 0 & $0.0(0.0 ; 11.9)$ & 0 & $0.0(0.0 ; 5.5)$ & - \\
\hline Wound dehiscence & 0 & $0.0(0.0 ; 9.7)$ & 0 & $0.0(0.0 ; 11.9)$ & 0 & $0.0(0.0 ; 5.5)$ & - \\
\hline Hematoma (requiring revision) & 1 & $2.8(0.1 ; 14.5)$ & 0 & $0.0(0.0 ; 11.9)$ & 1 & $1.5(0.0 ; 8.3)$ & - \\
\hline Other wound/local tissue-related AE & 0 & $0.0(0.0 ; 9.7)$ & 0 & $0.0(0.0 ; 11.9)$ & 0 & $0.0(0.0 ; 5.5)$ & - \\
\hline Thromboembolic complications & 0 & $0.0(0.0 ; 9.7)$ & 0 & $0.0(0.0 ; 11.9)$ & 0 & $0.0(0.0 ; 5.5)$ & - \\
\hline Sepsis & 0 & $0.0(0.0 ; 9.7)$ & 0 & $0.0(0.0 ; 11.9)$ & 0 & $0.0(0.0 ; 5.5)$ & - \\
\hline Pneumonia & 0 & $0.0(0.0 ; 9.7)$ & 0 & $0.0(0.0 ; 11.9)$ & 0 & $0.0(0.0 ; 5.5)$ & - \\
\hline Renal insufficiency & 0 & $0.0(0.0 ; 9.7)$ & 0 & $0.0(0.0 ; 11.9)$ & 0 & $0.0(0.0 ; 5.5)$ & - \\
\hline Stroke & 0 & $0.0(0.0 ; 9.7)$ & 1 & $3.4(0.1 ; 17.8)$ & 1 & $1.5(0.0 ; 8.3)$ & - \\
\hline Sudden death & 0 & $0.0(0.0 ; 9.7)$ & 0 & $0.0(0.0 ; 11.9)$ & 0 & $0.0(0.0 ; 5.5)$ & - \\
\hline Other systemic $\mathrm{AE}$ & 4 & $11.1(3.1 ; 26.1)$ & 3 & $10.3(2.2 ; 27.4)$ & 7 & $10.8(4.4 ; 20.9)$ & - \\
\hline
\end{tabular}


Table 6 (continued)

The safety population was defined as all patients that have received PHILOS type of plate fixation. Patients were grouped according to the actual treatment they received. The two patients that received prothesis as the primary fixation are excluded in this table. Control group: PHILOS without augmentation. Augmented group: PHILOS with augmentation

$A E$ adverse event, $P H I L O S$ proximal humerus internal locking system

${ }^{\text {a } O n l y ~ A E s ~ s t a r t i n g ~ u p ~ t o ~} \leq 425$ days after surgery, i.e., with an onset before the upper visit window of the 1 -year follow-up, were included in the table

${ }^{b}$ Number of patients with at least one AE. If patient experienced multiple AEs under any complication class, the patient was only counted once

${ }^{\mathrm{c}}$ Estimated risk of developing at least one AE (calculated by dividing the number of patients experiencing at least one complication by the total number of patients in the corresponding treatment group. For this table, all the patients irrespective of availability of follow-up counted in the denominator)

${ }^{\mathrm{d}}$ Confidence intervals for percentages were calculated using the Clopper-Pearson method

${ }^{\mathrm{e}}$ Fisher's exact test

period of 1 year, while the previous study recorded radiological results at 3 months postoperatively. Ultimately, both studies were rather small and a larger prospective study with sufficient statistical power may help resolve the differences. One surprising outcome was the much lower than expected mechanical failure rate. With the highest mean age $(76.8 \pm 6.8)$ recorded in the PHF surgery literature so far $[15,27]$ and a very low BMD of $87.2 \pm 20[17,48]$, the current study nevertheless achieved an overall mechanical failure rate of around $15 \%$. Although it is difficult to compare our mechanical failure rate with literature values due to the reporting heterogeneity, the current mechanical failure rate is likely to be on the lower end of what have been reported $[4,14,20,23,27,49]$. In osteoporotic fracture care, with the restoration of the medial hinge and intrinsic stability through anatomical reduction, implants become a load sharing rather than a load bearing construct leading to reduced mechanical failure rates. Therefore, one possible explanation for the current low mechanical failure rate could be the improvement of osteoporotic fracture care in the past decade: better anatomical reduction through surgical means resulting in the restoration of intrinsic stability. In comparison to a previous study with a younger study population where anatomical reduction was achieved in $25.7 \%(9 / 35)$ of the patients and medial support achieved in 51.4\% (18/35), the current study had a much higher rate of anatomical reduction (68.6\% for the control group and $69 \%$ for the augmented group) and restoration of medial support $(62.9 \%$ for the control group and $62.1 \%$ for the augmented group) [7]. This is consistent with the previous suggestion that the most important factor in determining good outcomes was good surgical techniques [7, 11, 14, 17, 49]. In addition, a Hawthorne effect cannot be ruled out: given the prospective study design, the surgeons may have paid more attention to surgical details contributing to the observed lower mechanical failure rate.

Although it is hard to compare the AE rate of different studies due to their heterogeneity (e.g., different reporting criterion, study design, patient population, and different surgical techniques), the currently reported $\mathrm{AE}$ rate of $41.7 \%$ in the control group and $41.5 \%$ in the augmented group are in the upper range of previous reports $[5,9,10,14,20,22,49]$. This could be due to the prospective nature of the study and a more comprehensive collection of AEs. Consistent to a previous report that no additional complications were noted in screw augmentation in PHF [27], in the current study, only one patient suffered an $\mathrm{AE}$ related to the used Traumacem $\mathrm{V}+$ cement, i.e., intraoperative cement leakage into the shoulder joint. After a joint arthrotomy, the patient fully recovered. No allergic reaction to the Traumacem V+cement was reported.

Currently, it is common to perform primary reversed fracture arthroplasty in three- and four-part fractures with a cutoff age of 75 years [50, 51]. For complex fractures, this procedure is performed in patients aged 65 years and up (surgeon's observation). Our result of a low mechanical failure rate reported in both the control and the augmented groups would suggest that good outcome can be achieved in elderly complex PHF patients with low BMD treated with locking plates, and that a further lowering the age for primary reversed fracture arthroplasty may not be reasonable.

\section{Limitations}

The major limitation of the current study is its premature termination and a much smaller patient population than the original plan. For this reason, the study did not have the statistical power to detect differences between the study groups.

\section{Future direction}

Due to the difficulty in patient recruitment in the current study and that past RCTs have not resulted in sufficient evidence to recommend a treatment of choice, we anticipate that future RCTs are likely to encounter similar problems [52-54]. Prospective observational studies and registries are more likely to generate data from sufficiently large patient populations and are the recommended way to move the field forward. In light of the ongoing debate in the treatment protocol for complex PHF, i.e., arthroplasty versus 
osteosynthesis, nail versus plates, and conservative versus surgical, an observational approach also has the benefit of comparing multiple methods in one study.

\section{Conclusion}

Due to premature termination resulting in an underpowered study, evidence was not sufficient to show any differences between the two treatment groups regarding the risk of mechanical failure. However, considering that no additional risks related to the use of cement were observed, and that low mechanical failure rate was achieved in a population with high mean age and low BMD, PHILOS ${ }^{\mathrm{TM}}$ seems a good treatment option for elderly PHF in patients. Further studies are necessary to establish the effectiveness of screw-tip cement augmentation of PHILOS.

Acknowledgements We would like to thank AO Clinical Investigation and Documentation (AOCID) for conducting the study, including site management, medical and scientific support, statistical analysis, and medical writing services.

Funding The presented clinical investigation was performed with the support of the AO Foundation via the AOTK Trauma and the AOTrauma networks. Open access funding provided by University of Innsbruck and Medical University of Innsbruck.

\section{Compliance with ethical standards}

Conflict of interest Franz Kralinger is a consultant with DePuySynthes but did not receive personal benefits for the current study. Tim Pohlemann was the chairman of the AO TK-System during the time of study. Martin Jaeger is a member of the AO UEEG.

Research involving human participants and/or animals All the procedures performed in studies involving human participants were in accordance with the ethical standards of the institutional and/or national research committee, and with the 1964 Helsinki Declaration and its later amendments or comparable ethical standards

Informed consent Informed consent was obtained from all the individual participants included in the study.

OpenAccess This article is distributed under the terms of the Creative Commons Attribution 4.0 International License (http://creativeco mmons.org/licenses/by/4.0/), which permits unrestricted use, distribution, and reproduction in any medium, provided you give appropriate credit to the original author(s) and the source, provide a link to the Creative Commons license, and indicate if changes were made.

\section{References}

1. Bahrs C, Stojicevic T, Blumenstock G, Brorson S, Badke A, Stockle U, Rolauffs B, Freude T (2014) Trends in epidemiology and pathoanatomical pattern of proximal humeral fractures. Int Orthop 38(8):1697-1704. https://doi.org/10.1007/s00264-014-2362-6
2. Voigt $\mathrm{C}$, Lill H (2011) [Innovations and prognoses]. Unfallchirurg 114(12):1083-1090. https://doi.org/10.1007/s00113-011-2056-0

3. Agudelo J, Schurmann M, Stahel P, Helwig P, Morgan SJ, Zechel W, Bahrs C, Parekh A, Ziran B, Williams A, Smith W (2007) Analysis of efficacy and failure in proximal humerus fractures treated with locking plates. J Orthop Trauma 21(10):676-681. https://doi.org/10.1097/BOT.0b013e31815bb09d

4. Owsley KC, Gorczyca JT (2008) Fracture displacement and screw cutout after open reduction and locked plate fixation of proximal humeral fractures [corrected]. J Bone Joint Surg Am 90(2):233240. https://doi.org/10.2106/JBJS.F.01351

5. Egol KA, Ong CC, Walsh M, Jazrawi LM, Tejwani NC, Zuckerman JD (2008) Early complications in proximal humerus fractures (OTA Types 11) treated with locked plates. J Orthop Trauma 22(3):159-164. https://doi.org/10.1097/BOT.0b013e318169ef2a

6. Fankhauser F, Boldin C, Schippinger G, Haunschmid C, Szyszkowitz R (2005) A new locking plate for unstable fractures of the proximal humerus. Clin Orthop Relat Res (430):176-181

7. Gardner MJ, Weil Y, Barker JU, Kelly BT, Helfet DL, Lorich DG (2007) The importance of medial support in locked plating of proximal humerus fractures. J Orthop Trauma 21(3):185-191. https://doi.org/10.1097/BOT.0b013e3180333094

8. Solberg BD, Moon CN, Franco DP, Paiement GD (2009) Locked plating of 3- and 4-part proximal humerus fractures in older patients: the effect of initial fracture pattern on outcome. J Orthop Trauma 23(2):113-119. https://doi.org/10.1097/BOT.0b013e3181 9344bf

9. Solberg BD, Moon CN, Franco DP, Paiement GD (2009) Surgical treatment of three and four-part proximal humeral fractures. J Bone Joint Surg Am 91(7):1689-1697. https://doi.org/10.2106/ JBJS.H.00133

10. Konrad G, Hirschmuller A, Audige L, Lambert S, Hertel R, Sudkamp NP (2012) Comparison of two different locking plates for two-, three- and four-part proximal humeral fractures-results of an international multicentre study. Int Orthop 36(5):1051-1058. https://doi.org/10.1007/s00264-011-1410-8

11. Erasmo R, Guerra G, Guerra L (2014) Fractures and fracture-dislocations of the proximal humerus: a retrospective analysis of 82 cases treated with the Philos((R)) locking plate. Injury 45(Suppl 6):S43-S48. https://doi.org/10.1016/j.injury.2014.10.022

12. Geiger EV, Maier M, Kelm A, Wutzler S, Seebach C, Marzi I (2010) Functional outcome and complications following PHILOS plate fixation in proximal humeral fractures. Acta Orthop Traumatol Turc 44(1):1-6. https://doi.org/10.3944/AOTT.2010.2270

13. Sproul RC, Iyengar JJ, Devcic Z, Feeley BT (2011) A systematic review of locking plate fixation of proximal humerus fractures. Injury 42(4):408-413. https://doi.org/10.1016/j.injur y.2010.11.058

14. Sudkamp N, Bayer J, Hepp P, Voigt C, Oestern H, Kaab M, Luo C, Plecko M, Wendt K, Kostler W, Konrad G (2009) Open reduction and internal fixation of proximal humeral fractures with use of the locking proximal humerus plate. Results of a prospective, multicenter, observational study. J Bone Joint Surg Am 91(6):13201328. https://doi.org/10.2106/JBJS.H.00006

15. Thanasas C, Kontakis G, Angoules A, Limb D, Giannoudis P (2009) Treatment of proximal humerus fractures with locking plates: a systematic review. J Shoulder Elbow Surg 18(6):837844. https://doi.org/10.1016/j.jse.2009.06.004

16. Clavert P, Adam P, Bevort A, Bonnomet F, Kempf JF (2010) Pitfalls and complications with locking plate for proximal humerus fracture. J Shoulder Elbow Surg 19(4):489-494. https://doi. org/10.1016/j.jse.2009.09.005

17. Krappinger D, Bizzotto N, Riedmann S, Kammerlander C, Hengg C, Kralinger FS (2011) Predicting failure after surgical fixation of proximal humerus fractures. Injury 42(11):1283-1288. https:// doi.org/10.1016/j.injury.2011.01.017 
18. Gardner MJ, Lorich DG, Werner CM, Helfet DL (2007) Secondgeneration concepts for locked plating of proximal humerus fractures. Am J Orthop (Belle Mead NJ) 36(9):460-465

19. Murray IR, Amin AK, White TO, Robinson CM (2011) Proximal humeral fractures: current concepts in classification, treatment and outcomes. J Bone Joint Surg Br 93(1):1-11. https://doi. org/10.1302/0301-620X.93B1.25702

20. Brunner F, Sommer C, Bahrs C, Heuwinkel R, Hafner C, Rillmann P, Kohut G, Ekelund A, Muller M, Audige L, Babst R (2009) Open reduction and internal fixation of proximal humerus fractures using a proximal humeral locked plate: a prospective multicenter analysis. J Orthop Trauma 23(3):163-172. https://doi. org/10.1097/BOT.0b013e3181920e5b

21. Kammerlander C, Neuerburg C, Verlaan JJ, Schmoelz W, Miclau T, Larsson S (2016) The use of augmentation techniques in osteoporotic fracture fixation. Injury 47(Suppl 2):S36-S43. https://doi. org/10.1016/s0020-1383(16)47007-5

22. Egol KA, Sugi MT, Ong CC, Montero N, Davidovitch R, Zuckerman JD (2012) Fracture site augmentation with calcium phosphate cement reduces screw penetration after open reduction-internal fixation of proximal humeral fractures. J Shoulder Elbow Surg 21(6):741-748. https://doi.org/10.1016/j.jse.2011.09.017

23. Kralinger F, Blauth M, Goldhahn J, Kach K, Voigt C, Platz A, Hanson B (2014) The influence of local bone density on the outcome of one hundred and fifty proximal humeral fractures treated with a locking plate. J Bone Joint Surg Am 96(12):1026-1032. https://doi.org/10.2106/JBJS.M.00028

24. Unger S, Erhart S, Kralinger F, Blauth M, Schmoelz W (2012) The effect of in situ augmentation on implant anchorage in proximal humeral head fractures. Injury 43(10):1759-1763. https://doi. org/10.1016/j.injury.2012.07.003

25. Kathrein S, Kralinger F, Blauth M, Schmoelz W (2013) Biomechanical comparison of an angular stable plate with augmented and non-augmented screws in a newly developed shoulder test bench. Clin Biomech (Bristol Avon) 28(3):273-277. https://doi. org/10.1016/j.clinbiomech.2012.12.013

26. Roderer G, Scola A, Schmolz W, Gebhard F, Windolf M, Hofmann-Fliri L (2013) Biomechanical in vitro assessment of screw augmentation in locked plating of proximal humerus fractures. Injury 44(10):1327-1332. https://doi.org/10.1016/j.injur y.2013.05.008

27. Katthagen JC, Lutz O, Voigt C, Lill H, Ellwein A (2018) Cement augmentation of humeral head screws reduces early implant-related complications after locked plating of proximal humeral fractures. Obere Extremitat 13(2):123-129. https://doi. org/10.1007/s11678-018-0440-x

28. Schliemann B, Wahnert D, Theisen C, Herbort M, Kosters C, Raschke MJ, Weimann A (2015) How to enhance the stability of locking plate fixation of proximal humerus fractures? An overview of current biomechanical and clinical data. Injury 46(7):12071214. https://doi.org/10.1016/j.injury.2015.04.020

29. Laux CJ, Grubhofer F, Werner CML, Simmen HP, Osterhoff G (2017) Current concepts in locking plate fixation of proximal humerus fractures. J Orthop Surg Res 12(1):137. https://doi. org/10.1186/s13018-017-0639-3

30. Harris PA, Taylor R, Thielke R, Payne J, Gonzalez N, Conde JG (2009) Research electronic data capture (REDCap)-a metadatadriven methodology and workflow process for providing translational research informatics support. J Biomed Inf 42(2):377-381. https://doi.org/10.1016/j.jbi.2008.08.010

31. DePuySymthes (2016) PHILOS and PHILOS long. DePuy Synthes Trauma, a division of Synthes $\mathrm{GmbH}$, West Chester

32. DePuySynthes (2015) PHILOS with Augmentation Surgical Technique. DePuy Synthes Trauma, a division of Synthes GmbH, West Chester
33. Krappinger D, Roth T, Gschwentner M, Suckert A, Blauth M, Hengg C, Kralinger F (2012) Preoperative assessment of the cancellous bone mineral density of the proximal humerus using CT data. Skeletal Radiol 41(3):299-304. https://doi.org/10.1007/ s00256-011-1174-7

34. Charlson M, Szatrowski TP, Peterson J, Gold J (1994) Validation of a combined comorbidity index. J Clin Epidemiol 47(11):1245-1251

35. Charlson ME, Pompei P, Ales KL, MacKenzie CR (1987) A new method of classifying prognostic comorbidity in longitudinal studies: development and validation. J Chronic Dis 40(5):373-383

36. Beaton DE, Wright JG, Katz JN, Upper Extremity Collaborative G (2005) Development of the QuickDASH: comparison of three item-reduction approaches. J Bone Joint Surg Am 87(5):10381046. https://doi.org/10.2106/JBJS.D.02060

37. Gummesson C, Ward MM, Atroshi I (2006) The shortened disabilities of the arm, shoulder and hand questionnaire (QuickDASH): validity and reliability based on responses within the full-length DASH. BMC Musculoskelet Disord 7:44. https://doi. org/10.1186/1471-2474-7-44

38. Hudak PL, Amadio PC, Bombardier C (1996) Development of an upper extremity outcome measure: the DASH (disabilities of the arm, shoulder and hand) [corrected]. The Upper Extremity Collaborative Group (UECG). Am J Ind Med 29 (6):602-608. https://doi.org/10.1002/(SICI)1097-0274(199606)29:6\%3C602 :AID-AJIM4\%3E3.0.CO;2-L

39. Bot SD, Terwee CB, van der Windt DA, Bouter LM, Dekker J, de Vet HC (2004) Clinimetric evaluation of shoulder disability questionnaires: a systematic review of the literature. Ann Rheum Dis 63(4):335-341

40. MacDermid JC (1996) Development of a scale for patient rating of wrist pain and disability. J Hand Ther 9(2):178-183

41. MacDermid JC, Solomon P, Prkachin K (2006) The Shoulder Pain and Disability Index demonstrates factor, construct and longitudinal validity. BMC Musculoskelet Disord 7:12. https://doi. org/10.1186/1471-2474-7-12

42. Constant CR (1991) [Assessment of shoulder function]. Orthopade 20(5):289-294

43. Constant CR, Murley AH (1987) A clinical method of functional assessment of the shoulder. Clin Orthop Relat Res 214:160-164

44. Neer CS $2^{\text {nd }}(1970)$ Displaced proximal humeral fractures. I. Classification and evaluation. J Bone Joint Surg Am 52(6):1077-1089

45. Somasundaram K, Huber CP, Babu V, Zadeh H (2013) Proximal humeral fractures: the role of calcium sulphate augmentation and extended deltoid splitting approach in internal fixation using locking plates. Injury 44(4):481-487. https://doi.org/10.1016/j.injur y.2012.10.030

46. Gracitelli ME, Malavolta EA, Assuncao JH, Kojima KE, dos Reis PR, Silva JS, Ferreira Neto AA, Hernandez AJ (2016) Locking intramedullary nails compared with locking plates for two- and three-part proximal humeral surgical neck fractures: a randomized controlled trial. J Shoulder Elbow Surg 25(5):695-703. https://doi. org/10.1016/j.jse.2016.02.003

47. Kloub M, Holub K, Polakova S (2014) Nailing of three- and four-part fractures of the humeral head-long-term results. Injury 45(Suppl 1):S29-S37. https://doi.org/10.1016/j.injur y.2013.10.038

48. American College of Radiology (2018) ACR-SPR-SSR practice parameter for the performance of musculoskeletal quantitative computed tomography (QCT)

49. Beeres FJP, Hallensleben NDL, Rhemrev SJ, Goslings JC, Oehme F, Meylaerts SAG, Babst R, Schep NWL (2017) Plate fixation of the proximal humerus: an international multicentre comparative study of postoperative complications. Arch Orthop Trauma Surg 137(12):1685-1692. https://doi.org/10.1007/s00402-017-2790-Z 
50. Smith GC, Bateman E, Cass B, Damiani M, Harper W, Jones H, Lieu D, Petchell J, Petrelis M, Piper K, Sher D, Smithers CJ, Trantalis J, Vrancic S, Harris IA (2017) Reverse Shoulder Arthroplasty for the treatment of Proximal humeral fractures in the Elderly (ReShAPE trial): study protocol for a multicentre combined randomised controlled and observational trial. Trials 18(1):91. https ://doi.org/10.1186/s13063-017-1826-6

51. Anakwenze OA, Zoller S, Ahmad CS, Levine WN (2014) Reverse shoulder arthroplasty for acute proximal humerus fractures: a systematic review. J Shoulder Elbow Surg 23(4):e73-e80. https://doi. org/10.1016/j.jse.2013.09.012

52. Schumaier A, Grawe B (2018) Proximal humerus fractures: evaluation and management in the elderly patient. Geriatr Orthop Surg Rehabil 9:2151458517750516. https://doi.org/10.1177/21514 58517750516
53. Li M, Wang Y, Zhang Y, Yang M, Zhang P, Jiang B (2018) Intramedullary nail versus locking plate for treatment of proximal humeral fractures: a meta-analysis based on 1384 individuals. J Int Med Res 46(11):4363-4376. https://doi.org/10.1177/0300060518 781666

54. Sun Q, Ge W, Li G, Wu J, Lu G, Cai M, Li S (2018) Locking plates versus intramedullary nails in the management of displaced proximal humeral fractures: a systematic review and meta-analysis. Int Orthop 42(3):641-650. https://doi.org/10.1007/s0026 4-017-3683-z

Publisher's Note Springer Nature remains neutral with regard to jurisdictional claims in published maps and institutional affiliations. 